\title{
DESCRIPCIÓN DE CINCO ESPECIES NUEVAS DE CERION (MOLLUSCA: PULMONATA: CERIONIDAE) DE LA REGIÓN ORIENTAL DE CUBA
}

\section{Description of five new species of Cerion (Mollusca: Pulmonata: Cerionidae) from the eastern cuban region}

\begin{abstract}
Alexis Suárez ${ }^{1, *}$ y Alejandro Fernández ${ }^{2}$
${ }^{1}$ Sociedad Cubana de Zoología. Carretera de Varona 11875 e/ Oriente y Lindero. Habana 19. Calabazar. Cuba. 11900. (1D) orcid.org/0000-0002-0623-8808. ${ }^{2}$ Centro de Investigaciones y Servicios Ambientales y Tecnológicos, CISATCITMA-Holguín. (1) orcid.org/0000-0002-4419-2353. "Para correspondencia: alexys02@nauta.cu.
\end{abstract}

\section{RESUMEN}

Se describen cinco especies nuevas del género Cerion Röding, 1878, procedentes cuatro de ellas de la provincia de Holguín, y una de la provincia de Guantánamo, aumentando a 156 las especies y subespecies descritas de Cuba. Como caracteres diagnósticos notables se mencionan para Cerion orteai sp. nov., con localidad tipo en Playa Baracutey, la presencia de dientes columelares y un diente parietal comparativamente más elevado; para Cerion naylae sp. nov., con localidad tipo en Playa Carmona, la ausencia de costillas en la mitad de la concha; para Cerion espinosai sp. nov., con localidad tipo en Playa Baracutey, la forma escalonada que adopta la concha en la zona de inserción sutural; para Cerion moralespradoi $\mathbf{s p . ~ n o v . , ~ c o n ~ l o c a l i d a d ~ t i p o ~ e n ~}$ El Canto, un ápice redondeado y diente parietal bilobulado; y para Cerion harasewychi sp. nov., con localidad tipo en Rafael Freyre, la superficie de la concha con costillas tenues apenas perceptibles. Para todas estas especies se incluyen datos de anatomía interna asociados a la rádula y órganos reproductores.

Palabras clave: Cerion orteai, Cerion naylae, Cerion espinosai, Cerion moralespradoi, Cerion harasewychi, Cuba.

\section{ABSTRACT}

Five new species from the genus Cerion Roding, 1878 (Gastropoda: Cerionidae) are described, been four of them from Holguín province, and one from Guantanamo province, increasing to 156 the number of species described for Cuba. As notable diagnostic characters are mentioned for Cerion orteai sp. nov., with type locality in Playa Baracutey, Ramón de Antillas, the presence of columellar teeth and a comparative taller parietal tooth; for Cerion naylae sp. nov., with type locality in Playa Carmona, Ramón de Antillas, the absence of ribs in the half of the shell; for Cerion espinosai sp. nov., with type locality in Playa Baracutey, Ramón de Antillas, the stair way adopted during insertion in suture; for Cerion moralespradoi sp. nov., with type locality in El Canto, Punta de Maisí, a rounded apex, and a bilobulate parietal tooth; and for Cerion harasewychi sp. nov., with type locality in Rafael Freyre, Gibara, a conch surface with unperceptible ribs. For all these species, data of radule and reproductive organs are given. 


\section{INTRODUCCIÓN}

Hasta hace relativamente poco tiempo, del género Cerion Röding, 1798, se conocían en Cuba 147 formas, entre especies y subespecies (Espinosa y Ortea, 2009), al que en trabajos recientes, le son incorporadas cuatro más (Suárez, 2015, 2018, 2019), tres de ellas para la provincia de Holguín (Cerion milerai Suárez, 2018; Cerion alejandroi Suárez, 2019, y Cerion seguii, Suárez, 2019), y una de la provincia de Guantánamo (Cerion petreus Suárez, 2015), incrementándose el total 151 formas.

En la provincia de Holguín, este grupo se encuentra bien representado, actualmente con 38 formas descritas, entre especies y subespecies, las que muestran un marcado endemismo, carácter que hace a estas poblaciones vulnerables a factores derivados de la antropización, como la fragmentación, destrucción y degradación de los ecosistemas (Espinosa y Ortea, 2009; Fernández et al., 2015, 2016).

Los ceriónidos habitan zonas costeras con hábitats empobrecidos (Clench y Aguayo, 1952), y condiciones extremas (García et al., 2017), donde imperan el calor excesivo y alta salinidad (Espinosa y Ortea, 2009).

\section{OBJETIVO}

- Describir cinco nuevas especies del género Cerion Röding, 1798.

\section{MATERIALES Y MÉTODOS}

Los ejemplares utilizados para la descripción de estas nuevas especies fueron recolectados durante el trabajo de campo. Las medidas de la concha se tomaron siguiendo la técnica propuesta por Aguayo y Jaume (1944), utilizando un calibrador digital Powerfix.

Para la obtención de las partes blandas se siguió el método utilizado por Suárez (2018). Las muestras se sometieron a disección bajo microscopio estereoscópico a 20X aumentos, con la ayuda de pinzas de disección, obteniéndose de esta manera el aparato reproductor. Las rádulas fueron obtenidas utilizando la técnica propuesta por Clench (1924-1925). Para el ordenamiento taxonómico de la especie se utilizó el propuesto por Uit de Weerd (2008). Todas las medidas son dadas en milímetros. Para la identificación de los órganos del aparato reproductor se hizo uso de las descripciones y diagramas que se muestran en Batsch (1920) y Shileyko (1999).

Los actos nomenclaturales de esta publicación han sido registrados en ZooBank, sistema de registro en línea de la Comisión Internacional de Nomenclatura Zoológica. La información asociada a los identificadores registrados (LSID) podrá ser visualizada a través de cualquier navegador web, agregando el LSID al prefijo "http://zoobank.org/". El LSID para esta publicación es: urn: 1sid:zoobank.org:pub:AB01770E-8883-4197-A90B-FB59369D01B5. Cada especie aparece con su identificador particular.

Abreviaturas. IES: Instituto de Ecología y Sistemática. 


\author{
RESULTADOS \\ TAXONOMÍA \\ Clase GASTROPODA Cuvier, 1795 \\ Subclase PULMONATA Cuvier, 1814 \\ Orden STYLOMMATOPHORA Schmidt, 1856 \\ Superfamilia: Urocoptoidea Uit de Weerd, 2008 \\ Familia CERIONIDAE Pilsbry, 1901 \\ Género Cerion Röding, 1798 \\ Subgénero Strophiops Dall, 1894 \\ Cerion (Strophiops) orteai sp. nov.
}

(Figuras 1, 2)

Material examinado. Se utilizaron las conchas de 9 ejemplares adultos de C. orteai sp. nov., recolectados en agosto de 2018.

Diagnosis. Concha pequeña, con vueltas abultadas, lados rectos, y costillas anchas; ápice cónico, abertura peristomal ovalada con dientes columelares observados con dificultad hacia el interior de la concha, y uno parietal más elevado, peristoma fino y ombligo estrecho. Cerion orteai sp. nov., se diferencia de Cerion milerai Suárez, 2018, por poseer una concha más pequeña, un diente parietal más elevado, y un peristoma más fino; de Cerion pygmaeum Pilsbry y Vanatta, 1896, por poseer vueltas de lados más rectos, vueltas más voluminosas, el ápice más cónico y las costillas más anchas, y de Cerion saetiae Sánchez Roig, 1948, por poseer su superficie acostillada, con vueltas abultadas, abertura peristomal ovalada y ombligo más estrecho; además, Cerion orteai sp. nov., se diferencia de las anteriores por la presencia de dientes columelares.

Diagnosis (in English). Shell of small size, with bulging whorls, straight sides, and wide ribs; a conic apex and oval aperture with internal columellar teeth, watched with some difficulty, and one taller parietal tooth; thick peristome and narrow navel. Cerion orteai sp. nov., it differs from Cerion milerai Suárez, 2018, by the smaller size of the conch, a higher peristomal tooth, and a thicker peristome; from Cerion pygmaeum Pilsbry y Vanatta, 1896, by having straighter sides, bulging whorls, a more conic apex and wider ribs; from Cerion saetiae Sánchez Roig, 1948, by having a ribbed surface, bulging whorls, oval aperture and narrower navel. Cerion orteai sp. nov., it also differs from all anterior mentioned species by the presence of columellar teeth.

Descripción del Holotipo. Concha mediana de color ambarino, forma ligeramente alargada, de $91 / 2$ vueltas, acostillada; longitud mayor 20.04; longitud menor $18.47 \mathrm{~mm}$; diámetro mayor $9.64 \mathrm{~mm}$; diámetro menor $9.58 \mathrm{~mm}$; diámetro mayor de la abertura peristomal $9.07 \mathrm{~mm}$; diámetro menor de la abertura peristomal $6.94 \mathrm{~mm}$ (Fig. 1). Ápice de base ancha, corto y puntiagudo, de $4.01 \mathrm{~mm}$ con $7 \frac{1}{2}$ vueltas escalonadas en la inserción a la sutura, poseyendo un núcleo amplio de una vuelta, elevado y liso, de color ambarino, al que le sigue la teleoconcha de $1 \frac{1}{4}$ vueltas, finamente acostillada, dándole paso a las vueltas restantes (seis vueltas) en el ápice. Las vueltas que le siguen, hasta llegar a la abertura peristomal estrecha de la concha, son redondeadas, mientras que en la región apical son lisas. Costillas de base ancha, elevadas y romas, espacio intercostal similar al ancho de las costillas. La última vuelta con 18 costillas que se adentran en el ombligo, el que es amplio y profundo. Peristoma expandido de borde fino, en forma oval, reflejado hacia atrás. Diente parietal alto de base ancha y dos dientes columelares bajos de base ancha (Fig. 2), alejados de la apertura. Interior de la concha blanco. 


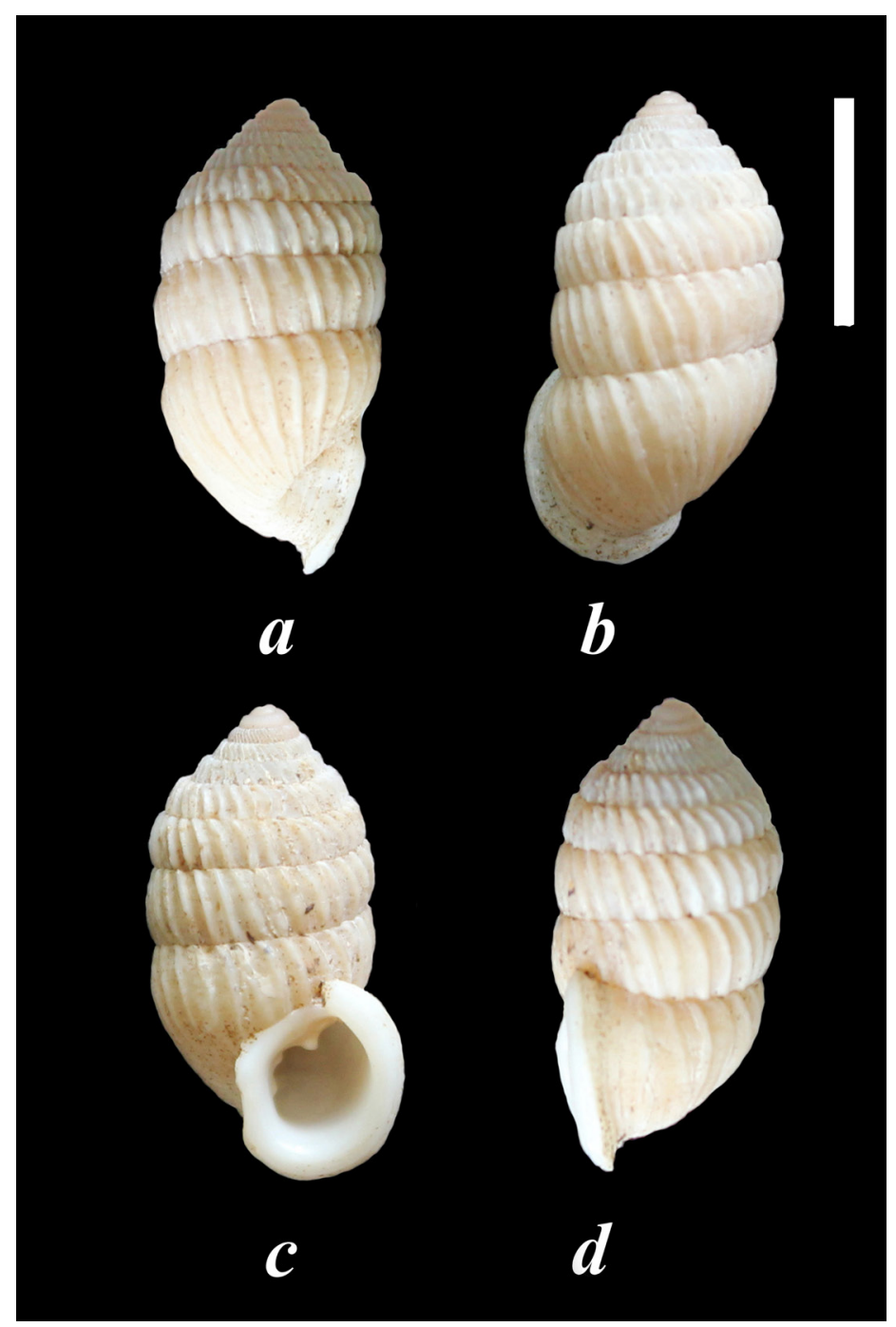

Figura 1. Holotipo de Cerion orteai sp. nov. Vista lateral derecha (a); vista superior (b); vista inferior (c); vista lateral izquierda (d). Escala $10 \mathrm{~mm}$.

Anatomía del Holotipo. Rádula de $1.200 \mathrm{~mm}$ de largo y $1.057 \mathrm{~mm}$ de ancho; fórmula radular 23-1-23; largo del diente central $0.021 \mathrm{~mm}$; ancho del diente central $0.021 \mathrm{~mm}$. El divertículo de la bursa copulatrix en el sistema reproductor es de $18.65 \mathrm{~mm}$, la distancia entre el borde externo del atrium hasta la inserción del epífalo en el prepucio es de $1.45 \mathrm{~mm}$; pene de $2.00 \mathrm{~mm}$.

Localidad tipo. Playa Baracutey, localidad de El Ramón de Antillas, Holguín, Cuba $\left(20^{\circ} 52^{\prime} \mathrm{N}\right.$; $\left.75^{\circ} 37^{\prime} \mathrm{O}\right)$.

Material tipo: Holotipo, Instituto de Ecología y Sistemática, Cuba: CZACC8. 1. 307., provenientes de la localidad tipo.

Distribución. Conocida solo de esta localidad.

Etimología. Especie dedicada a Jesús Ortea, investigador español que ha dedicado gran parte de su labor al estudio de la malacofauna cubana. 


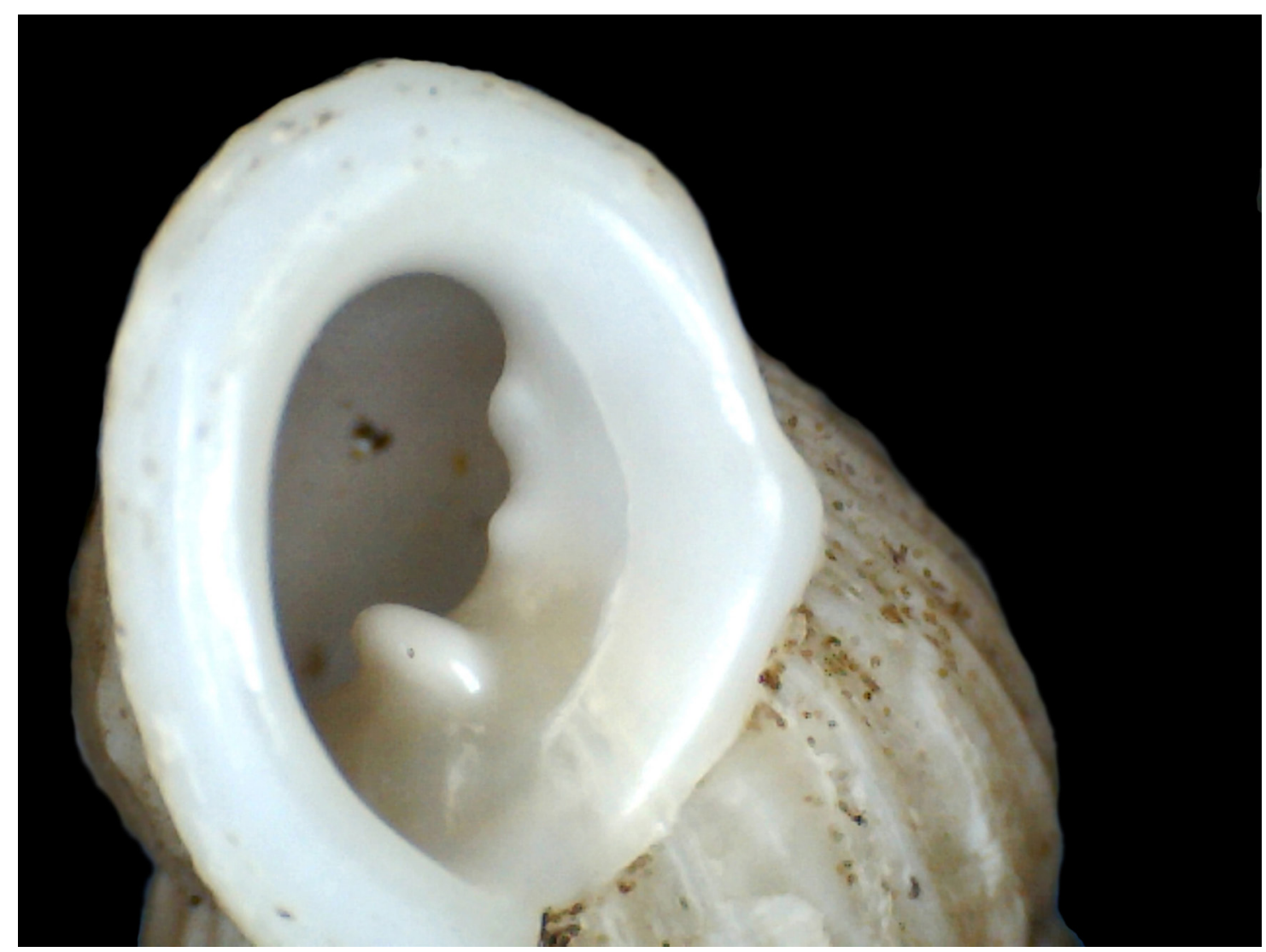

Figura 2. Apertura peristomal del Holotipo de Cerion orteai sp. nov., donde se observan un diente parietal y dos dientes columelares bajos.

Ecología. Ejemplares observados al borde del uveral y sobre el substrato rocoso que aflora en la superficie del terreno.

Variabilidad. En la población se hallan individuos cuyo diente columelar se halla bilobulado hacia el interior de la concha, en forma horizontal, a diferencia del holotipo, cuyos dientes columelares se hallan alineados horizontalmente, al igual que otros ejemplares con diente columelar muy bajo, casi ausente.

Urn: 1sid:zoobank.org:act:4a2d5c9a-2cd1-474a-9ced-a5254ff52593.

Cerion (Strophiops) naylae sp. nov.

(Figuras 3, 4)

Material examinado. Se utilizaron las conchas de 15 ejemplares adultos de C. naylae sp. nov., recolectados en agosto de 2018.

Diagnosis. Concha grande, alargada, cilíndrica de lados paralelos, ápice corto, tres últimas vueltas alargadas, núcleo bajo, estrecho y punticulado. Abertura peristomal amplia, con diente parietal pequeño, a diferencia del diente columelar, que es notable. Línea sutural en última vuelta que interrumpe el curso de las costillas hacia el ombligo. Ausencia de costillas en una de sus vueltas post apicales. Cerion naylae sp. nov., se diferencia de Cerion banesense Clench y Aguayo, 1949, por poseer una abertura peristomal más amplia, así como las tres últimas vueltas más cilíndricas; de Cerion blanesi Clench y Aguayo, 1951, por poseer una concha más robusta y 
de mayor tamaño; de Cerion caroli aedilii Aguayo y de la Torre, 1951, se diferencia por poseer una concha más corta, cilíndrica, con costillas menos manifiestas; de Cerion catherwoodianum Wurtz, 1950, por poseer mayor espacio intercostal, abertura peristomal menos alargada, y una forma más alargada y cilíndrica; de Cerion ceiba ceiba Clench, 1948, por poseer una concha no pupoide; de Cerion cisnerosi Clench y Aguayo, 1951, por poseer una concha más cilíndrica y menos acostillado; Cerion cobarrubia Aguayo y Jaume, 1951, por mostrar una concha menos pupoide y menor número de costillas; de Cerion dominicanum Clench y Aguayo, 1951, por mostrar los lados de la concha menos voluptuosos; de Cerion longidens Pilsbry, 1902, por su forma más cilíndrica; de Cerion manatiense Aguayo y Jaume, 1951, por poseer costillas oblicuas; de Cerion nipense Aguayo, 1953, por su forma cilíndrica y la inserción de las vueltas en la sutura de las región apical no sobresaliente; de Cerion politum maisianum Pilsbry, 1902, por tener menor número de costillas en la penúltima vuelta; y de Cerion saugeti Aguayo y Jaume, 1951, por poseer menor número de costillas en su última vuelta. De las especies anteriormente mencionadas se diferencia por la ausencia de costillas en una de las vueltas post apicales.

Diagnosis (in English). Shell of big size, elongated, cilindrical with parallel sides; short apex, with a low and narrow nucleus having very small holds, like points. Three last whorls enlarged; wide aperture with small parietal teeth and notable columellar teeth. Sutural line on last whorl interrupting the ribs course to the navel letting them to get into the navel. Cerion naylae sp. nov., it differs from Cerion banesense Clench \& Aguayo, 1949, by having a wider aperture, and the three last whorls more cilindrical; from Cerion blanesi Clench \& Aguayo, 1951, by having a more robust shell and bigger in size; from Cerion catherwoodianum Wurtz, 1950, by the wider inter-ribs space, a less elongated aperture, and a more elongated and cilindrical form; Cerion caroli aedilii Aguayo \& de la Torre, 1951, it differs by having a shorter shell, cilindrical form with less manifest ribs; from Cerion ceiba ceiba Clench, 1948, by having a not pupa form; from Cerion cisnerosi Clench \& Aguayo, 1951, by having a more cilindrical shell with less ribs; from Cerion cobarrubia Aguayo \& Jaume, 1951, by having a less pupa shell and less ribs; from Cerion dominicanum Clench \& Aguayo, 1951, by showing less thick sides; from Cerion longidens Pilsbry, 1902, by the more cilindrical form; from Cerion manatiense Aguayo \& Jaume, 1951, by having oblique ribs; from Cerion mumiola Pfeiffer, 1839, by having a cilindrical shell with straight sides and a less swelled peristome; from Cerion nipense Aguayo, 1953, by the cilindrical form of the shell and insertion of whorls in suture of apical region with not projected character; from Cerion politum maisianum Pilsbry, 1902, by having less ribs through penultimate whorl; from Cerion saugeti Aguayo \& Jaume, 1951, by having less ribs through last whorl.

Descripción del Holotipo. Concha grande, de 10 1/2 vueltas, acostillada, de forma cilíndrica y color ambarino; longitud mayor $31.36 \mathrm{~mm}$; longitud menor $29.99 \mathrm{~mm}$; diámetro mayor $12.77 \mathrm{~mm}$; diámetro menor $12.74 \mathrm{~mm}$; diámetro mayor de la abertura peristomal $11.67 \mathrm{~mm}$; diámetro menor de la abertura peristomal $10.27 \mathrm{~mm}$ (Fig. 3). Ápice corto, de base ancha, cónico, pero no muy aguzado, de $7.18 \mathrm{~mm}$ con siete vueltas, poseyendo un núcleo bajo, punticulado al observarse con aumentos, de color rosado, más bien estrecho, de una vuelta, al que le sigue la teleoconcha de vuelta y media, de color rosado, finamente acostillada, dándole paso a las primeras vueltas de la concha, de coloración parda rosácea, en las que se hacen más notables las costillas. A partir de la séptima vuelta la concha se torna cilíndrica, hasta llegar al peristoma, el que es amplio, redondeado, con borde parietal más bien fino, liso y reflejado hacia atrás, con borde filoso. En la última vuelta solo aparecen costillas en la mitad superior de esta, ya que son interrumpidas por una tenue línea sutural, impidiendo que estas continúen su trayectoria sin adentrarse en el ombligo, el que es aquillado y abierto. Diente parietal pequeño pero perceptible, no muy expuesto. Diente columelar notable, hacia el interior. Interior de la concha con ligero tinte pardo amarillento. 
Anatomía del Holotipo. Rádula de $3.410 \mathrm{~mm}$ de largo y $0.725 \mathrm{~mm}$ de ancho; fórmula radular 24-1-24, largo del diente central $0.021 \mathrm{~mm}$; ancho del diente central $0.021 \mathrm{~mm}$. El divertículo de la bursa copulatrix en el sistema reproductor es de $3.030 \mathrm{~mm}$, la distancia entre el borde externo del atrium hasta la inserción del epífalo en el prepucio es de $2.352 \mathrm{~mm}$; pene de $3.251 \mathrm{~mm}$.

Material tipo. Holotipo, Instituto de Ecología y Sistemática, Cuba: CZACC8. 1. 308., provenientes de la localidad tipo.

Localidad tipo. Playa Carmona, El Ramón de Antillas, Holguín, Cuba (2049Nㅜ 75³4’ O).

Distribución. Conocida solo de esta localidad.

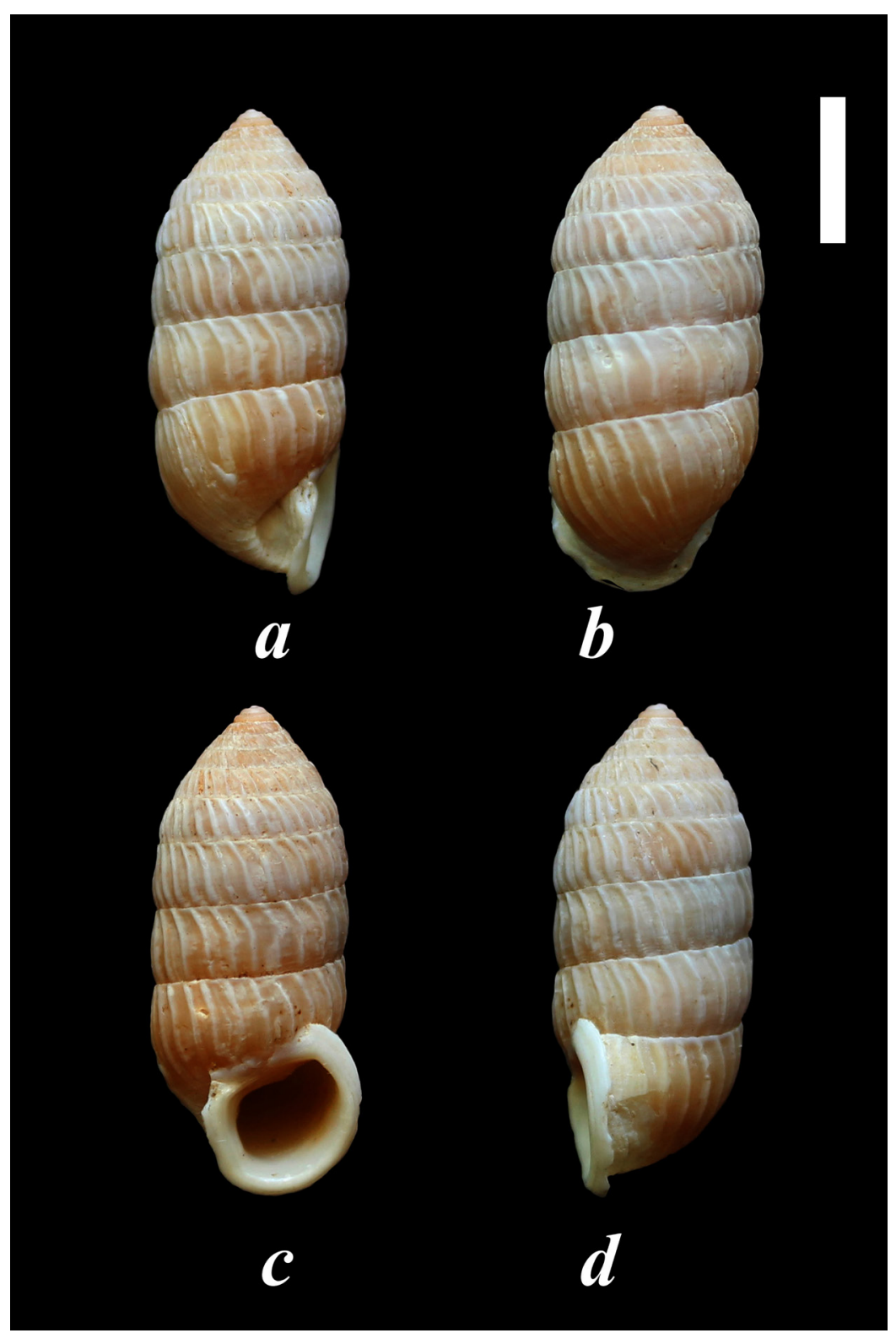

Figura 3. Holotipo de Cerion naylae sp. nov. Vista lateral derecha (a); vista superior (b); vista inferior (c); vista lateral izquierda (d). Escala $10 \mathrm{~mm}$. 


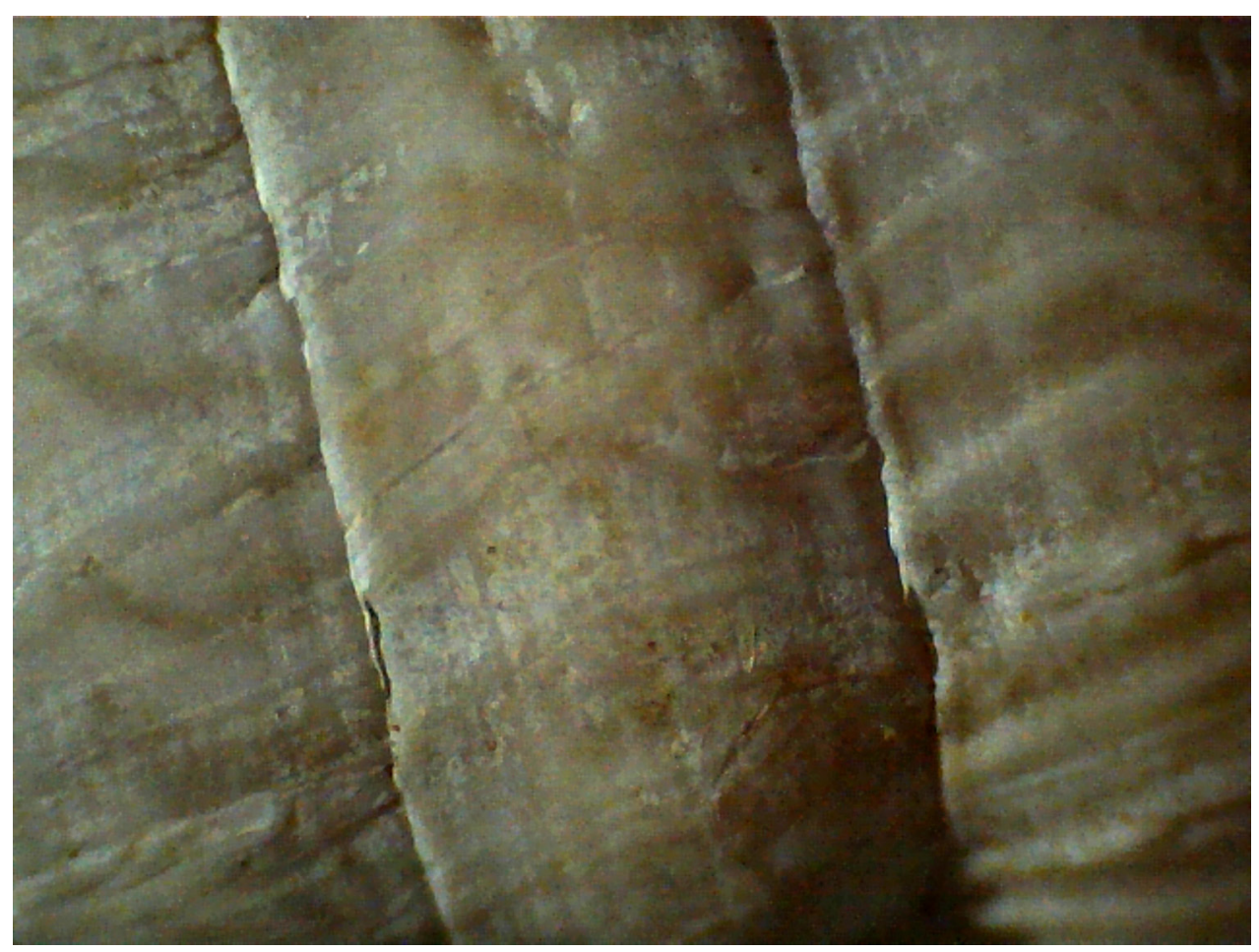

Figura 4. Detalle de la concha de un ejemplar de Cerion naylae sp. nov., donde se aprecia la variabilidad con ausencia de costillas.

Etimología. Dedicado a Nayla García Hernández, investigadora de la que he recibido un gran e incondicional apoyo durante el transcurso de investigaciones.

Variación. En la población aparecen ejemplares cuyo número de costillas es variable en la porción central de las conchas (Fig. 4), poseyendo espacios donde son apenas perceptibles, además de haber otros que no poseen un total desarrollo del cayo parietal.

Ecología. En senderos internos al borde del uveral, detrás de la duna de arena.

Comentario. En la colección malacológica del Museo de Zoología de Hamburgo, Alemania, aparecen ejemplares recolectados en el año 1957, con características conquiológicas y localidades similares, etiquetados como Cerion peninsularis, especie que nunca fue descrita, por lo que constituye un nomen nudum.

Urn:lsid:zoobank.org:act:82bf53f1-1e59-476a-94db-a499c3f206d3.

\section{Cerion (Strophiops) espinosai sp. nov.}

(Figuras 5,6)

Material examinado. Se utilizaron las conchas de 5 ejemplares adultos de C. espinosai sp. nov., recolectados en septiembre de 2017.

Diagnosis. Concha cilíndrica y alargada de pequeño tamaño, completamente acostillada, teniendo las costillas el mismo grosor que el espacio intercostal. Ápice con núcleo liso y alto, con una teleoconcha acostillada. Vueltas de la concha elevadas y proyectadas en la zona de unión sutural. Costillas en última vuelta menos notables cerca del peristoma, convirtiéndose en líneas axiales. Ombligo muy estrecho y cerrado. Cerion espinosai sp. nov., se diferencia de 
Cerion blanesi Clench y Aguayo, 1951, por la forma más fina de la concha, poseer un diente parietal más notable, y el color interno de la concha, siendo en Cerion espinosai sp. nov., de color oscuro. De Cerion ceiba canasiense Aguayo y de la Torre, 1951, se diferencia por poseer una concha más fina. De Cerion cisnerosi Clench y Aguayo, 1951, difiere por la forma más fina de la concha, poseyendo esta especie una concha de apariencia más globosa y costillas más notables, con una sutura menos notable y un diente columelar más notable. De Cerion ebriolum Aguayo y Jaume, 1951, se diferencia por poseer una concha más acostillada y más fina con lados menos redondeados, ya que $C$. ebriolum posee vueltas globosas, además de ambos dientes, columelar y parietal, más notables, con peristoma más grueso y callo parietal mejor formado. De Cerion macrodon Aguayo y Jaume, 1951, difiere por poseer una concha de lados más rectos, con interior pálido y peristoma más fino, con diente parietal menos notable. De Cerion incrassatum microdon Pilsbry y Vanatta, 1896, se diferencia porque esta posee una concha más redondeada hacia región posterior y más aguzada en la región anterior con abertura peristomal más redondeada; de Cerion pygmaeum Pilsbry y Vanatta, 1896, se diferencia por tener una forma cilíndrica más alargada, recta y no obesa, abertura peristomal más estrecha y alargada, color pálido del interior de la concha, y la transformación que sufren las costillas en la última vuelta; de Cerion tenuilabris Gundlach y Pfeiffer, 1870, difiere por el tamaño más corto, teniendo un cayo menos desarrollado y costillas más desarrolladas. De todas las especies anteriormente mencionadas, Cerion espinosai, sp. nov., se diferencia por poseer una concha en la que se muestra de forma muy notable la elevación de la concha en la zona de unión sutural sobre la vuelta anterior, donde el límite de las costillas sobresale por encima de la sutura.

Diagnosis (in English). Shell of small size with both, cylindrical and elongated form, completely ribbed, been ribs as wide as spaces among them. High and smooth nucleus in the apex, with a clearly ribbed teleoconch. High and projected whorls that mount on the suture of anterior whorls, where ribs termination are notables. Ribs in last whorl less notable near the peristome, locking like axial lines. Navel very narrow and sealed. Cerion espinosai sp. nov., it differs from Cerion pygmaeum Pilsbry \& Vanatta, 1896, by having a must cylindrical and large form, straight and not fat, peristome narrower and more elongated, pale color inside the conch and transformation that suffer ribs in the last whorl; from Cerion tenuilabris Gundlach \& Pfeiffer,1870, by the small size, having a less developed peristomal cay and more developed ribs; from Cerion blanesi Clench \& Aguayo, 1951, by the thinner form of the conch, by having a higher parietal tooth, and the internal color of the shell, been in Cerion espinosai sp. nov., of dark color; from Cerion ceiba canasiense Aguayo \& de la Torre, 1951, by having a thinner shell; from Cerion cisnerosi Clench \& Aguayo, 1951, by the thinner shape of the shell, having this species more notable ribs and columellar tooth, and a unnotable suture; from Cerion ebriolum Aguayo \& Jaume, 1951, by a very ribbed and thinner shell, with more rounded sides, because $C$. ebriolum looks like a barrel, besides having more notables columellar and parietal teeth, with thicker peristome and a better formed parietal cay; from Cerion macrodon Aguayo \& Jaume, 1951, by having a shell with more straight sides, pale internal color, thinner peristome and parietal tooth less notable. From Cerion incrassatum microdon Pilsbry \& Vanatta, 1896, it differs by having a rounder shell toward the posterior region with a rounder peristomal aperture, and a sharpened form in the apical portion; from Cerion incrassatum pygmaeum Pilsbry \& Vanatta, 1896, it differs by having a more elongated cilindric shape, straight and not obese, a narrow and elongated peristomal aperture, pale color into the shell, and the change that suffer the ribs on last whorl. From Cerion tenuilabris Gundlach \& Pfeiffer, 1870, it differs by the smaller size, having a less developed cay in aperture and more developed ribs. From all anterior mentioned species, Cerion espinosai sp. nov. differs by having a shell which exhibits a very notable elevation in sutural region on anterior whorl, where ribs limit protrudes over the suture. 
Descripción del Holotipo. Concha pequeña, de nueve vueltas, acostillada, de forma cilíndrico-alargada; longitud mayor $15.43 \mathrm{~mm}$; longitud menor $14.86 \mathrm{~mm}$; diámetro mayor $6.62 \mathrm{~mm}$; diámetro menor $6.59 \mathrm{~mm}$; diámetro mayor de la abertura peristomal $6.39 \mathrm{~mm}$; diámetro menor de la abertura peristomal $4.66 \mathrm{~mm}$ (Fig. 5). Ápice alargado y aguzado, proyectado, de $4.08 \mathrm{~mm}$ con $5 \mathrm{1} / 2$ vueltas, con núcleo elevado, amplio, de $1 \frac{1}{2} 2$ vueltas, liso, excepto en la zona de unión a la teleoconcha, donde se comienzan a ver muy pequeñas y superficiales líneas axiales, las que, al dar paso a la teleoconcha, de una vuelta, toman la apariencia de finas costillas algo más profundas, pero solo observadas con auxilio de una lente. Le siguen $6 \frac{1}{2} 2$ vueltas, (con $11.35 \mathrm{~mm}$ ) que forman el resto de la concha, cilíndrico alargado, las que se muestran notablemente elevadas cada una de ellas sobre la sutura de la vuelta anterior. Peristoma alargado, de borde ligeramente grueso, redondeado, con callo parietal poco notable. Diente parietal notable, fino. Diente columelar bajo pero perceptible. Interior de la concha pálido. Costillas elevadas, romas, con ligeras desviaciones, no rectas, siendo más notables en la zona de unión sutural correspondiente a la vuelta anterior, donde se observan escalonadas (Fig. 6.). En la última vuelta se cuentan 49 costillas en contacto con la sutura, ya que aparecen otras más finas (secundarias) un poco por debajo de la mitad, introduciéndose todas en el ombligo. Espacio intercostal del mismo ancho que las costillas. Ombligo alargado e inclinado, que se estrecha rápidamente hasta sellarse.

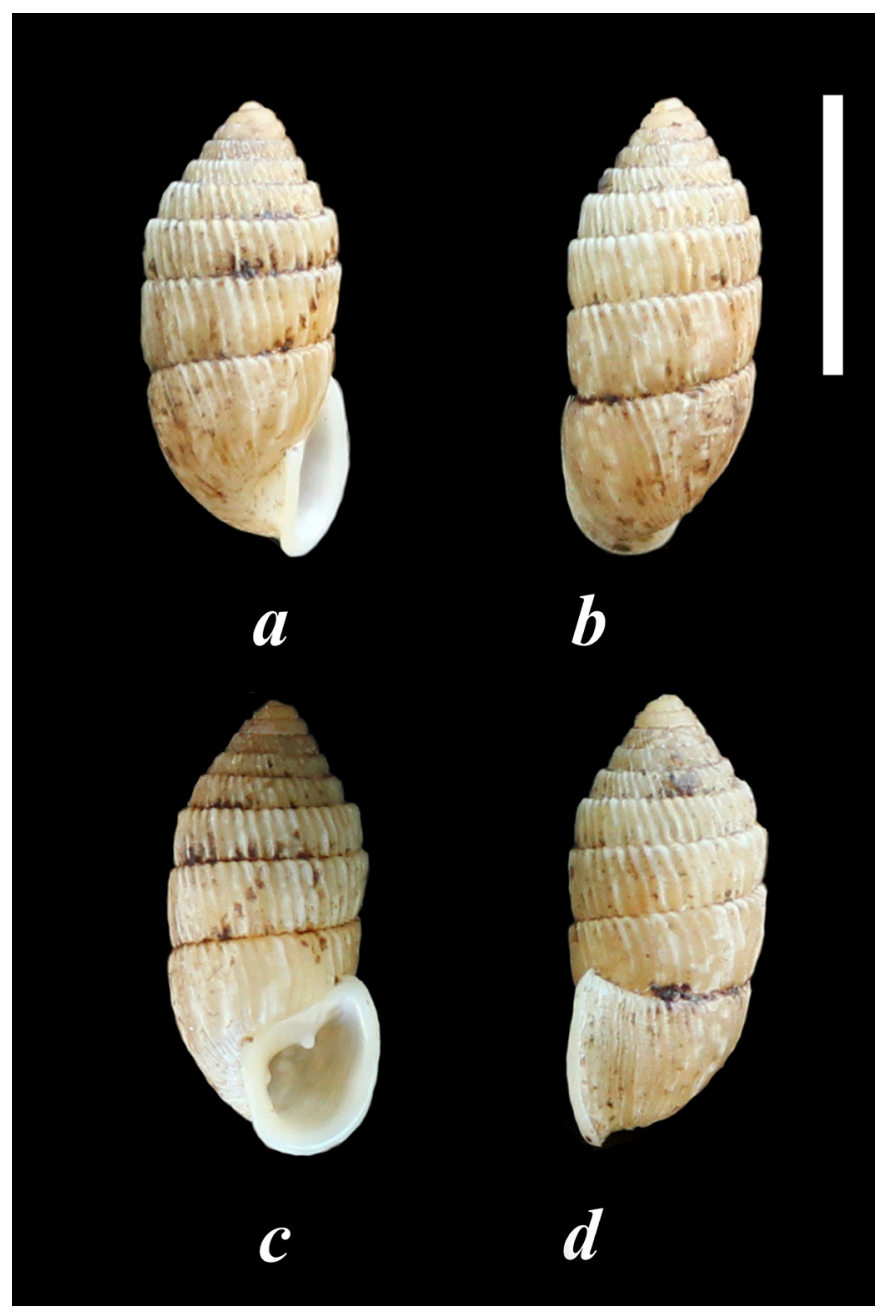

Figura 5. Holotipo de Cerion espinosai sp. nov.. Vista lateral derecha (a); vista superior (b); vista inferior (c); vista lateral izquierda (d). Escala $10 \mathrm{~mm}$. 


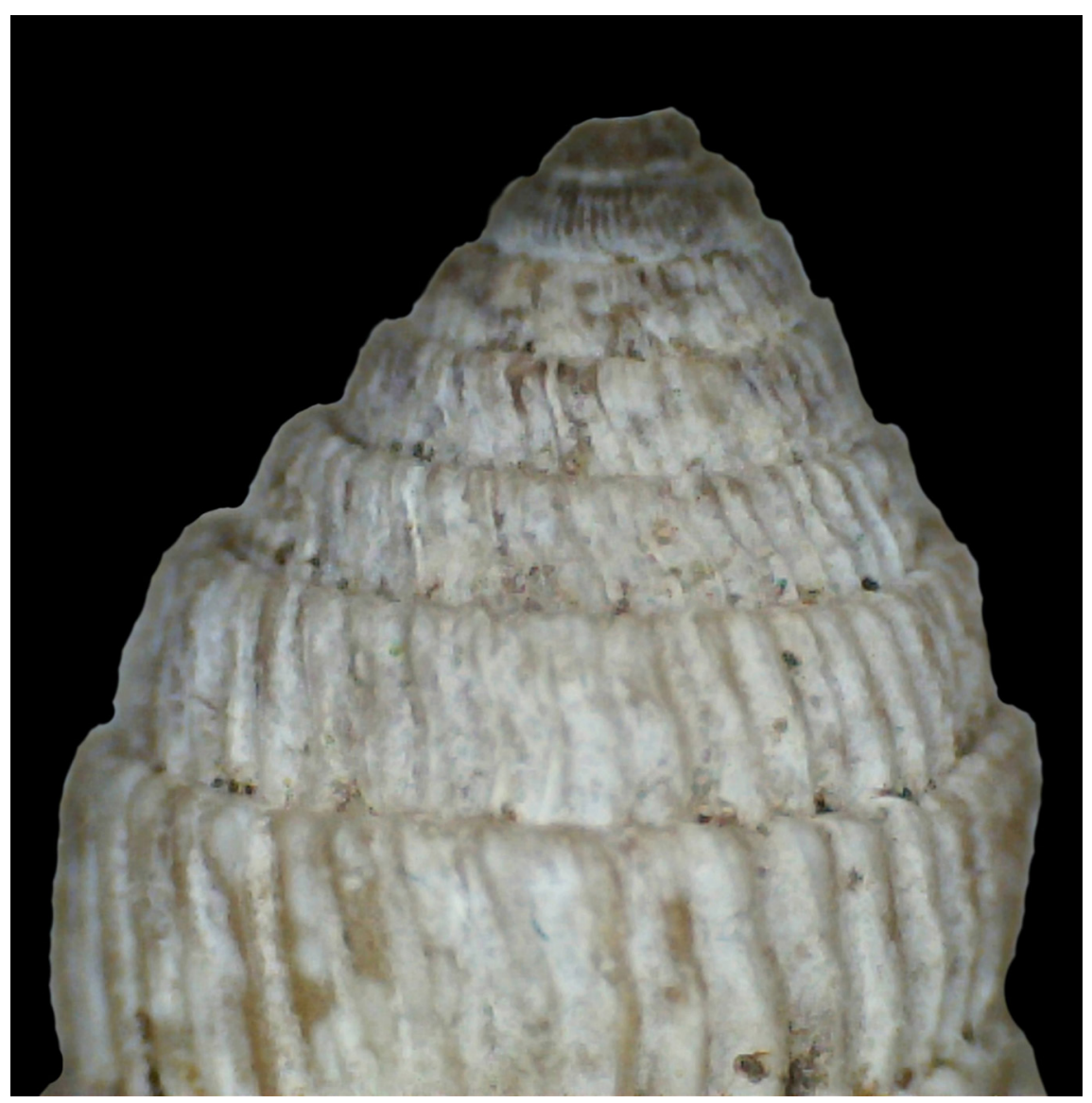

Figura 6. Detalle del Holotipo de Cerion espinosai sp. nov., donde se observa en la región sutural las vueltas elevadas, dándole forma escalonada.

Anatomía del Holotipo. Rádula de $2.350 \mathrm{~mm}$ de largo y $0.700 \mathrm{~mm}$ de ancho; fórmula radular 21-1-21, largo del diente central $0.014 \mathrm{~mm}$, ancho del diente central $0.015 \mathrm{~mm}$. El divertículo de la bursa copulatrix en el sistema reproductor es de $15.10 \mathrm{~mm}$, la distancia entre el borde externo del atrium hasta la inserción del epífalo en el prepucio es de $1.160 \mathrm{~mm}$; pene de $16.07 \mathrm{~mm}$.

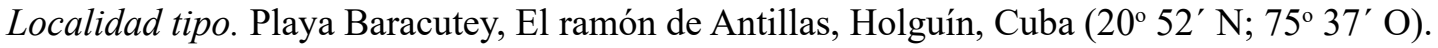

Material tipo. Holotipo, Instituto de Ecología y Sistemática, Cuba: CZACC8. 1.309, provenientes de la localidad tipo.

Distribución. Conocida solo de esta localidad.

Etimología. Especie dedicada a José Espinosa Sáenz, malacólogo cubano, comprometido con el estudio y la divulgación de nuestra malacofauna.

Ecología. Ejemplares recolectados hacia el interior del bosque siempreverde micrófilo en área sombreada y sobre plantas de Eugenia sp., Metopium toxiferum, y Thrinax radiata, a una altura sobre el suelo arenoso de entre $10 \mathrm{~cm}$ a $120 \mathrm{~cm}$. 


\section{Cerion (Strophiops) moralespradoi sp. nov.}

(Figuras 7, 8, 9)

Material examinado. Se utilizaron las conchas de 7 ejemplares adultos de C. moralespradoi sp. nov., recolectados en marzo de 2019.

Diagnosis. Concha mediana con ápice corto y redondeado; superficie lisa excepto en la última vuelta, donde aparecen costillas en la mitad inferior de la última vuelta, de la última vuelta, las que penetran en el ombligo; diente parietal bilobulado. Cerion moralespradoi sp. nov., se diferencia de Cerion lepidum Clench y Aguayo, 1951, por poseer una concha más pequeña, ápice redondeado, peristoma más fino y diente parietal bilobulado; de Cerion microdon Pilsbry y Vanatta, 1896, se diferencia por ser de mayor tamaño, poseer un ápice redondeado, diente parietal bilobulado, y superficie de la concha lisa. de Cerion marmorata polita Maynard, 1896, por poseer una concha de menor tamaño, peristoma fino y cortante, y un ápice más redondeado; de Cerion torrei Pilsbry y Vanatta, 1899, y Cerion torrei moralesi Clench y Aguayo, 1951, por poseer la concha más pequeña y estrecha, así como un diente parietal bilobulado; de Cerion politum maisianum Pilsbry, 1902, por no poseer costillas, sino una concha lisa, corta ymenos gruesa. Cerion moralespradoi sp. nov., se diferencia de todas las especies anteriormente mencionadas por poseer en la última vuelta costillas que se observan solo en la mitad inferior de la última vuelta.

Diagnosis (in English). Conch of medium size with both, short and rounded apex; Surface smooth except for the last whorl, where small ribs appear, been marked only from the half of the last whorl, approaching to the navel, and a bi-lobulated parietal tooth. Cerion moralespradoi sp. nov., it differs from Cerion lepidum Clench \& Aguayo, 1951, by having a smaller shell, a rounded apex, a thinner peristome with a bi-lobulated parietal tooth and a smooth surface; from Cerion microdon Pilsbry \& Vanatta, 1896, it differs for being bigger in size, a rounded apex, a thinner peristome with a bi-lobulated parietal tooth and a smooth surface; from Cerion marmorata polita Maynard, 1896, by having a smaller shell, a more rounded apex, both, a thinner and sharped peristome; from Cerion torrei torrei Pilsbry \& Vanatta, 1899, and Cerion torrei moralesi Clench \& Aguayo, 1951, because it has a thinner and narrower conch, as a bi-lobulated parietal tooth; from Cerion politum maisianum Pilsbry, 1902, for having a smooth surface, without ribs, besides showing a shorter and not fat shell. Of all these mentioned species Cerion moralespradoi sp. nov. differs by the ribs that has only in the inferior part of the conch in the last whorl.

Descripción del holotipo. Concha mediana, de nueve vueltas, de apariencia corta y ligeramente obesa, lisa excepto en las primeras vueltas postnucleares, con costillas bajas y muy apretadas, las que comprometen a la teleoconcha; longitud mayor $21.17 \mathrm{~mm}$; longitud menor; $19.81 \mathrm{~mm}$; diámetro mayor $10.75 \mathrm{~mm}$; diámetro menor $10.69 \mathrm{~mm}$; diámetro mayor de la abertura peristomal $9.68 \mathrm{~mm}$; diámetro menor de la abertura peristomal $7.91 \mathrm{~mm}$ (Fig. 7). Ápice corto y redondeado, no muy proyectado, de $4.46 \mathrm{~mm}$ con seis vueltas, con núcleo bajo, ancho y liso, de $1 \frac{1}{2}$ vueltas de color ámbar oscuro. Teleoconcha más estrecha que el núcleo, de una vuelta, color ambarino más tenue que el núcleo, acostillada siendo la vuelta más estrecha de la concha. Las siguientes tres vueltas con $16.17 \mathrm{~mm}$ son lisas, de color blanco cretácico con manchas alargadas esparcidas de color pardo claro. Abertura peristomal amplía con borde peristomal muy estrecho y filoso, sin proyección del callo parietal. Diente columelar alto, diente parietal amplio con una ligera ondulación (Fig. 8). Interior de la concha de color ambarino oscuro. Última vuelta lisa en la mitad superior, apareciendo en la mitad inferior costillas bajas (Fig. 9), que se adentran en el ombligo, el cual es profundo. 


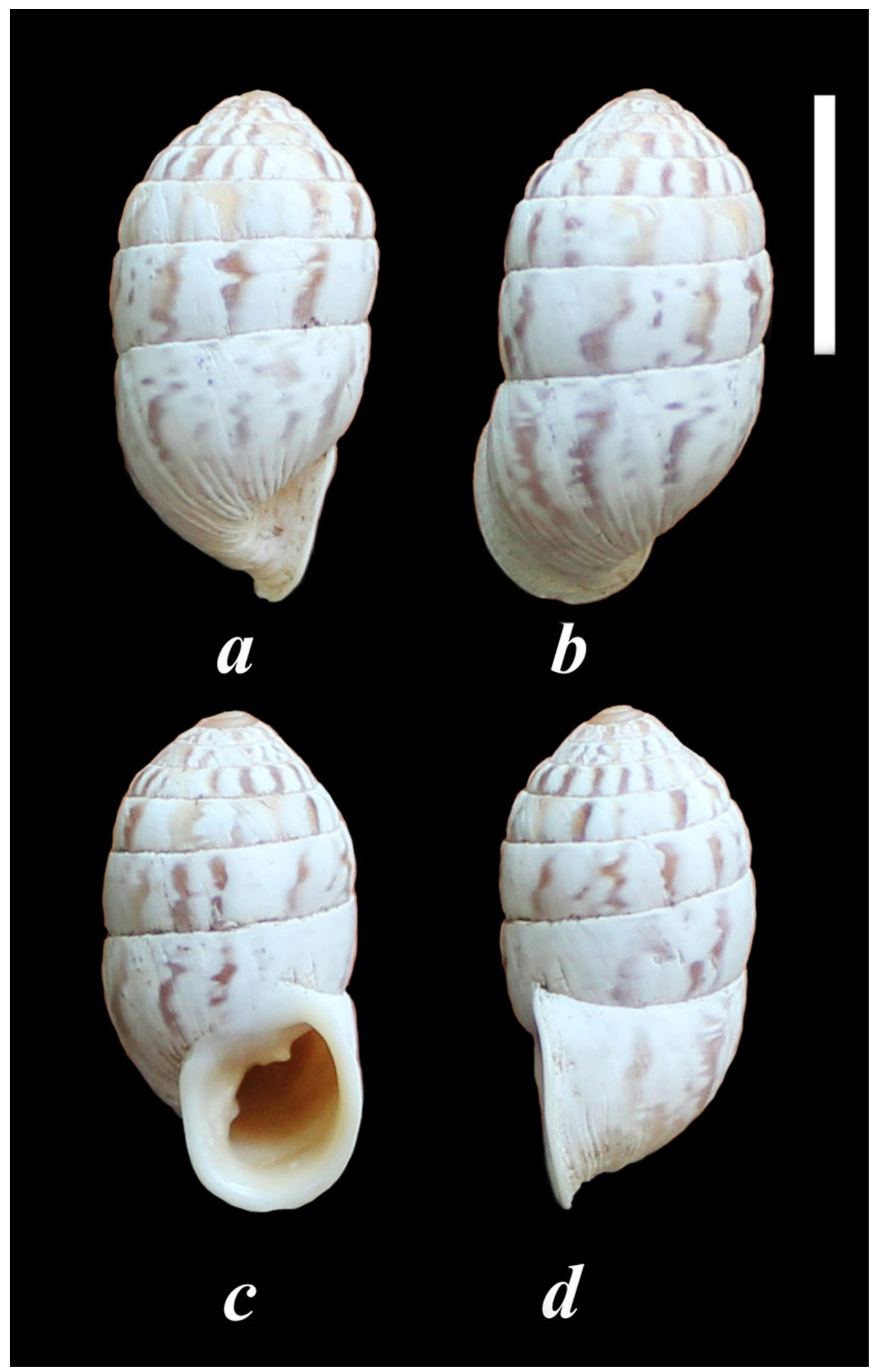

Figura 7. Holotipo de Cerion moralespradoi sp. nov. Vista lateral derecha (a); vista superior (b); vista inferior (c); vista lateral izquierda (d). Escala $10 \mathrm{~mm}$. 


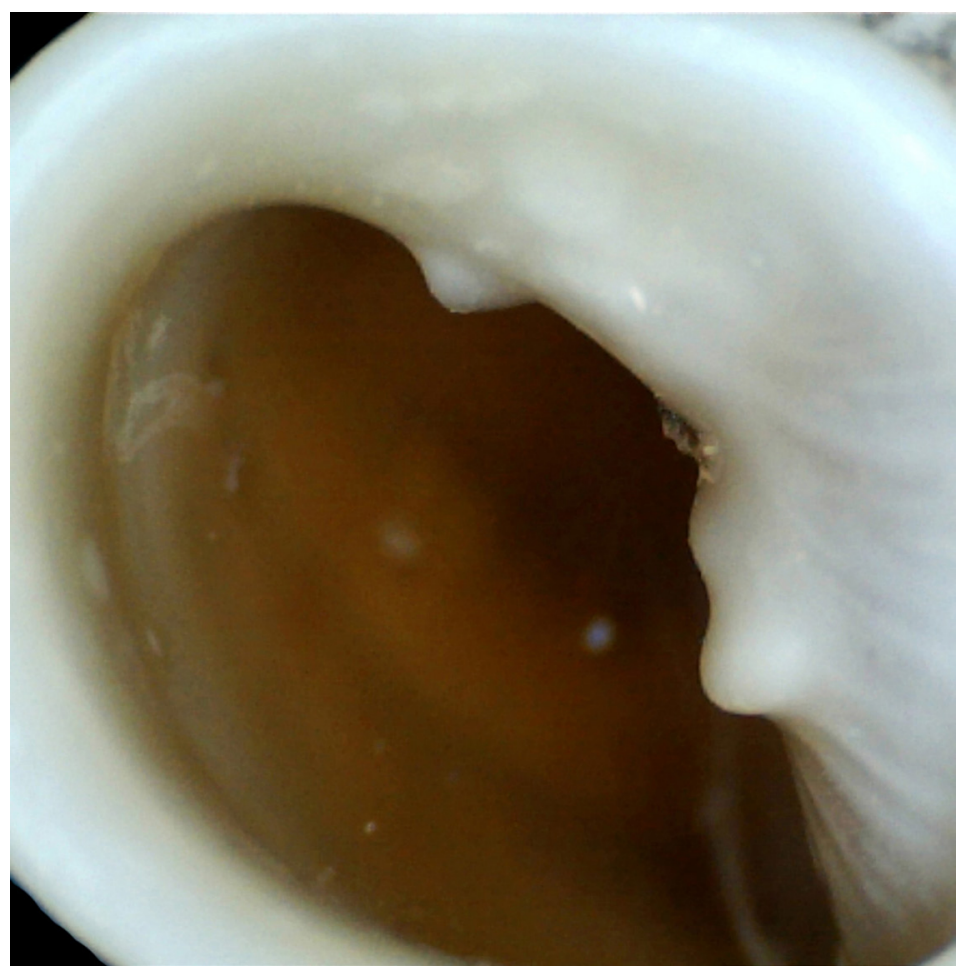

Figura 8. Detalle de la apertura de la concha Cerion moralespradoi sp. nov., donde se observa el diente parietal con ondulación.

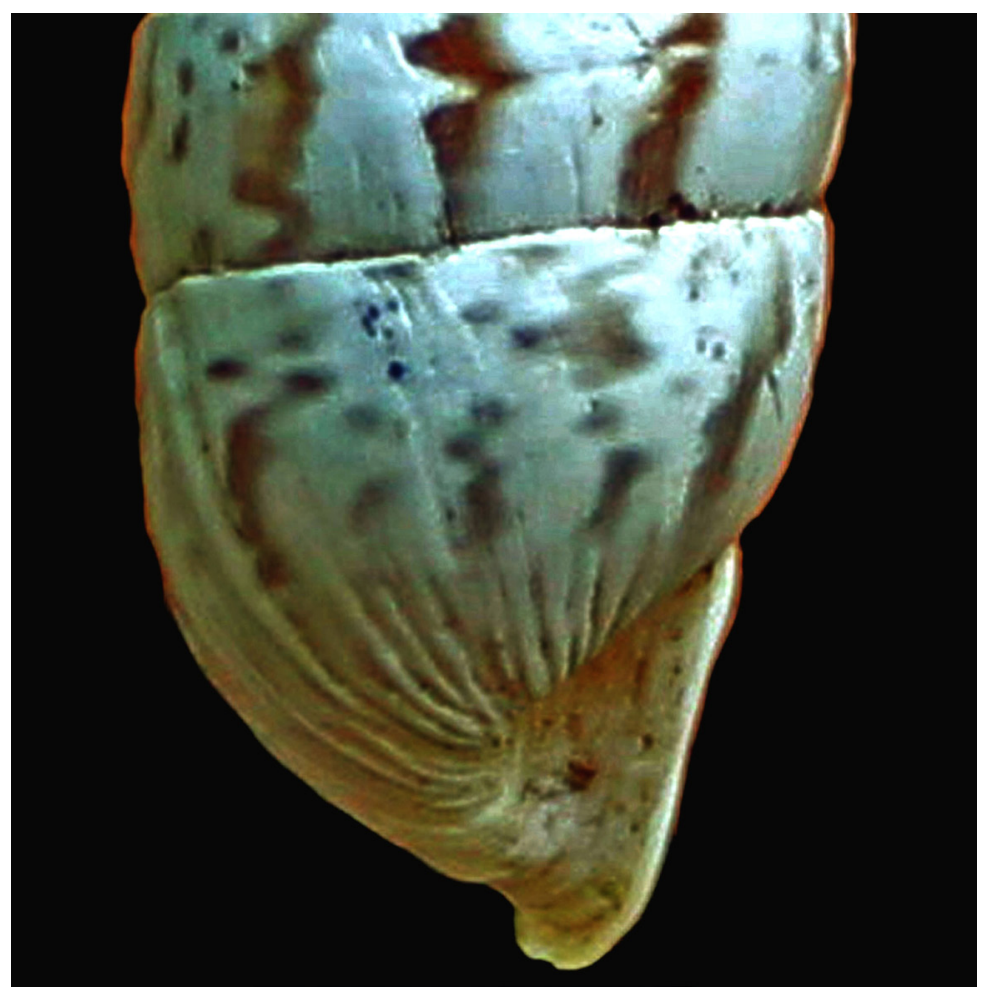

Figura 9. Detalle de la región posterior de Cerion moralespradoi sp. nov., donde se observa las costillas solo en la mitad inferior de la última vuelta. 
Anatomía del Holotipo. Rádula de $3.725 \mathrm{~mm}$ de largo y $0.990 \mathrm{~mm}$ de ancho; fórmula radular 24-1-24, largo del diente central $0.026 \mathrm{~mm}$, ancho del diente central $0.020 \mathrm{~mm}$. El divertículo de la bursa copulatrix en el sistema reproductor es de $19.99 \mathrm{~mm}$, la distancia entre el borde externo del atrium hasta la inserción del epífalo en el prepucio es de $1.549 \mathrm{~mm}$; pene de $2.150 \mathrm{~mm}$.

Material tipo. Holotipo, Instituto de Ecología y Sistemática, Cuba: CZACC8. 1.310, provenientes de la localidad tipo.

Localidad tipo. El Canto, Punta de Maisí, Guantánamo, Cuba (20 $17^{\prime} \mathrm{N} ; 7^{\circ} 1^{\prime}$ O); aproximadamente $6 \mathrm{~km}$ al Suroeste del faro.

Distribución. Conocida solo de esta localidad.

Etimología. Dedicado a Alexis Morales Prada, naturalista comprometido con la protección de los ecosistemas costeros en Punta de Maisí, Guantánamo.

Ecología. Ejemplares recolectados en vegetación espinosa baja, de unos $10 \mathrm{~cm}$ de altura del Matorral xeromorfo costero, detrás del Uveral, con exposición total al sol, aproximadamente a 90 metros de la línea de mareas.

Urn.1sid:zoobank.org:act:4924b797-aec4-4294-8f3f-f4d5b6b4e55b.

\section{Cerion (Strophiops) harasewychi sp. nov.}

(Figuras 10, 11)

Material examinado. Se utilizaron las conchas de 3 ejemplares adultos de $C$. harasewychi $\mathbf{s p . ~ n o v . , ~}$ recolectados en septiembre de 2018.

Diagnosis. Concha de pequeño tamaño y forma estrecha; con superficie lisa, costillas solo en las primeras vueltas, imperceptibles en las últimas, un diente parietal corto, y un delgado peristoma. Cerion harasewychi sp. nov., se diferencia de Cerion alleni Pilsbry, 1929, por su forma más pupoide y menor número de costillas; de Cerion ceiba minusculum Aguayo y A. de la Torre, 1952, por la forma pupoide y menor cantidad de costillas, por ser más estrecho y superficie de apariencia lisa; de Cerion coutini Sánchez Roig, 1951, por poseer una concha más estrecha y de menor tamaño, sin ornamentación y peristoma más estrecho; de Cerion crassiusculum Pilsbry y Vanatta, 1899, por su forma más estrecha, menor tamaño, menor número de costillas y tamaño del diente parietal; y de Cerion pygmaeum Pilsbry y Vanatta, 1896, por poseer una concha más estrecha, sin costillas pronunciadas ni numerosas.

Diagnosis (in English). Shell of small size and narrow form, with a smooth surface; ribs only in first whorls, imperceptibles in last; a short parietal tooth and a thin peristome. Cerion harasewychi sp. nov., it differs from Cerion alleni Pilsbry, 1929, by the more pupa form and less number of ribs in whorls; from Cerion ceiba minusculum Aguayo \& A. de la Torre, 1952, by the pupa form and having less ribs quantity, besides being narrower with a smoother surface; from Cerion coutini, Sánchez Roig, 1951, by having a narrower and smaller in size conch, with not ornaments, and a thinner peristome; Cerion crassiusculum Pilsbry \& Vanatta, 1899, because the narrower conch, smaller in size, less ribs quantity and the size of parietal tooth; from Cerion pygmaeum Pilsbry \& Vanatta, 1896, by having a narrower conch, without pronounced and numerous ribs. 
Descripción del Holotipo. Concha pequeña, alargada y estrecha, de 9 3/4 vueltas; longitud mayor 16.90; longitud menor $16.19 \mathrm{~mm}$; diámetro mayor $7.72 \mathrm{~mm}$; diámetro menor $7.64 \mathrm{~mm}$; diámetro mayor de la abertura peristomal $6.42 \mathrm{~mm}$; diámetro menor de la abertura peristomal $5.50 \mathrm{~mm}$ (Fig. 10). Protoconcha lisa, con núcleo estrecho y elevado. A partir de la teleoconcha, que es lisa, las vueltas del ápice son acostilladas, con costillas bajas e irregulares. Últimas dos vueltas lisas, de $9.21 \mathrm{~mm}$, con marcas axiales anchas que sustituyen a las costillas (Fig. 11). Ápice alargado, de $7.69 \mathrm{~mm}$ con siete vueltas. Abertura peristomal ligeramente oblicua, con peristoma estrecho pero notable. Diente columelar alto, diente parietal apenas perceptible. Ombligo alargado y oblicuo, que se estrecha rápidamente hasta cerrarse.

Anatomía del Holotipo. Rádula de $2.578 \mathrm{~mm}$ de largo y $0.841 \mathrm{~mm}$ de ancho; fórmula radular 23-1-23; largo del diente central $0.018 \mathrm{~mm}$; ancho del diente central $0.017 \mathrm{~mm}$. El divertículo de la bursa copulatrix en el sistema reproductor es de $16.35 \mathrm{~mm}$, la distancia entre el borde externo del atrium hasta la inserción del epífalo en el prepucio es de $1.200 \mathrm{~mm}$; pene de $1.709 \mathrm{~mm}$.

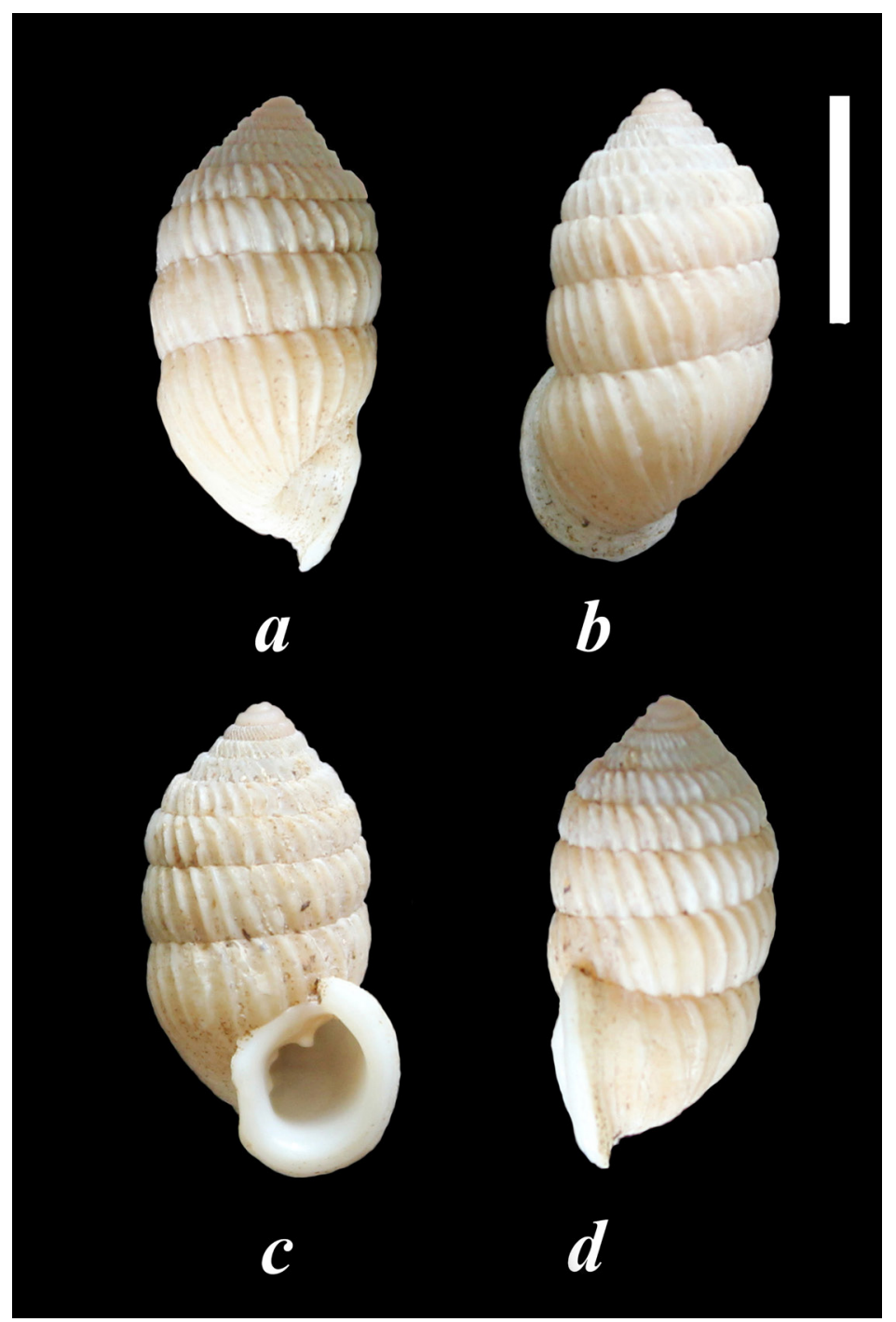

Figura 10. Holotipo de Cerion harasewychi sp. nov. Vista lateral derecha (a); vista superior (b); vista inferior (c); vista lateral izquierda (d). Escala $10 \mathrm{~mm}$. 


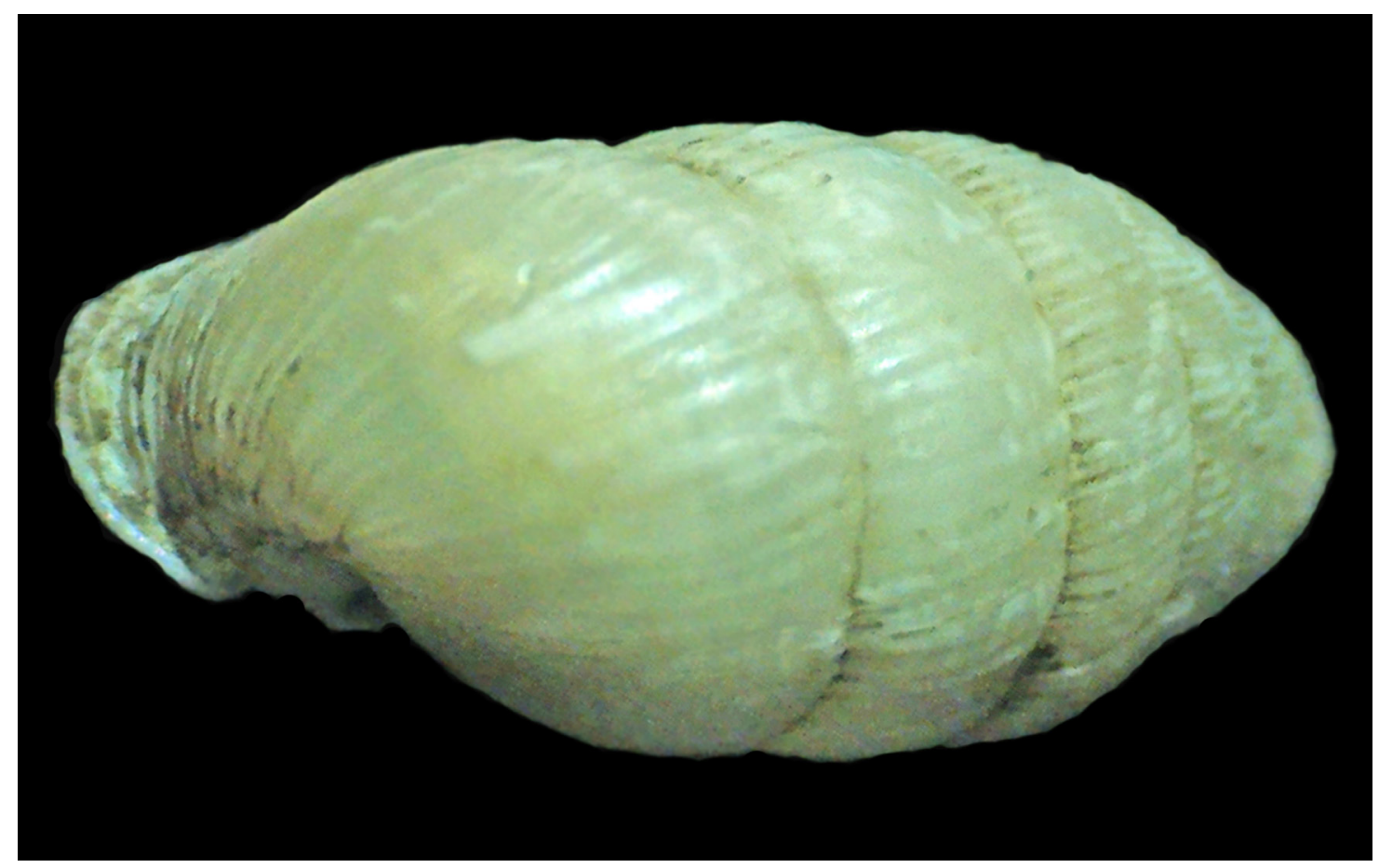

Figura 11. Holotipo de Cerion harasewychi sp. nov. Se observa la tendencia de las costillas a desaparecer en la última vuelta.

Material tipo. Holotipo, Instituto de Ecología y Sistemática, Cuba: CZACC8. 1. 311, recolectados en la localidad tipo.

Localidad tipo. Rafael Freyre, Gibara, Cuba (20 $52^{\prime}$; N; $\left.75^{\circ} 37^{\prime} \mathrm{O}\right)$.

Distribución. Conocida solo de esta localidad.

Etimología. Especie dedicada a Jerry Harasewych, estudioso de los ceriónidos, quien fuera Curador en el departamento de Zoología de los Invertebrados en el Museo Nacional de Historia Natural "Smithsonian Institution", por 30 años, actualmente Curador Emérito de dicha institución.

Ecología. Los Ejemplares estudiados fueron recolectados en la segunda terraza marina, de un metro de altura aproximadamente, detrás del manglar, en la zona del Yuraguanal.

Urn: lsid:zoobank.org:act:3e5939c5-24a1-4898-87c6-2d9f02fc95e2.

Nota General. Con la descripción de estas cinco nuevas especies, el inventario de los moluscos terrestres cubanos del género Cerion asciende a 156 formas entre especies y subespecies.

\section{AGRADECIMIENTOS}

El material descrito proviene de los muestreos como colaboración realizados en los proyectos "Representación cartográfica de información Geográfica y base de datos digital de la biodiversidad terrestre y marina en la zona norte oriental de sistema Cuba" y "Evaluación de los riesgos de la diversidad biológica en ecosistemas claves de la provincia Holguín", CISAT-CITMA". Agradecemos a Steffen Franke, Esteban Gutiérrez, José Espinosa y Luis De Armas, por la revisión crítica del manuscrito. 


\section{LITERATURA CITADA}

Aguayo, C. G. y M. L. Jaume. 1944. Guía para la descripción de moluscos gasterópodos. Revista de la Sociedad Malacológica Carlos de la Torre, 2 (2): 41-46.

Bartsch, P. 1920. Experiments in the breeding of Cerions. Department of Marine Biology of the Carnegie Institution of Washington. Vol: XIV, 106 pp.

Clench, W. J. 1924-1925. Radula technique for Physa. The Nautilus, 13: 13-14.

Clench, W. J. y C. G. Aguayo. 1952. The Scalarinum Species Complex (Umbonis) in the genus Cerion. Occ. Paper on Mollusks Museum of Comparative Zoology, 1 (17): 413-440.

Espinosa, J. y J. Ortea. 2009. Moluscos Terrestres de Cuba. Vaasa. Finlandia. 191 pp.

Fernández, A., A. Suárez y S. Franke. 2015. New records of rock dwelling molluscs at risk from tourist developments on coastal zone of Pesquero Nuevo, Holguín, Cuba. Tentacle, 23: $13-15$.

Fernández, A., S. Franke y A. Suárez. 2016. Restricted-range species in the coastal zone of Holguín, Cuba: Checklist and new records of priority species for conservation. Tentacle, 24: 7-10.

García, M, G., J. M. Martínez, M. I. Vinent, M. Hernández, P. M. Alcolado, B. M. Daranas, L. M. Cabrera, J. L. Juanes, C, Rodríguez, A. F. Márquez, M. A. Acosta y A. V. Núñez. 2017. Zonas costeras de Cuba. Importancia, protección y uso sostenible. $121 \mathrm{pp}$.

Shileyko, A. A. 1999. Treatise on recent terrestrial pulmonate molluscs. Draparnaudiidae, Caryodidae, Macrocyclidae, Acavidae, Clavatoridae, Dorcasiidae, Sculptariidae, Corillidae, Plectopylidae, Megalobulimidae, Strophocheilidae, Cerionidae, Achatinidae, Subulinidae, Glessulidae, Micractaeonidae, Ferrussaciidae. Ruthenica, Suplement 2, Part 4, 437-556.

Suárez, A. 2015. Nueva especie fósil de Cerion Röding, 1878 (Mollusca: Pulmonata: Cerionidae) de Cuba oriental. Novitates Caribaea, 8: 123-130.

Suárez, A. 2018. Especie nueva de Cerion (Mollusca: Pulmonata: Cerionidae) de Holguín, Cuba. Novitates Caribaea, 12: 43-48.

Suárez, A. 2019. Descripción de dos especies nuevas de Cerion (Mollusca: Pulmonata: Cerionidae) en estado subfósil, para Holguín, Cuba. Novitates Caribaea, 14: 121-127.

Uit de Weerd, D. R. 2008. Delimitation and phylogenetics of the highly diverse land snail family Urocoptidae (Gastropoda, Pulmonata) based on 28S rRNA sequence data: A reunion with Cerion. Journal of Molluscan Studies, 74: 317-329.

[Recibido: 5 de septiembre, 2019. Aceptado para publicación: 3 de mayo, 2020] 